\title{
SUM CONSTRUCTION OF AUTOMORPHIC BIBDS AND THEIR APPLICATIONS IN EXPERIMENTAL DESIGNS
}

\author{
G. S. DUGGAL ${ }^{1}$ and N. B. OKELO* \\ ${ }^{1}$ SCHOOL OF STATISTICS, UNIVERSITY OF GUJARAT, INDIA \\ *SCHOOL OF MATHEMATICS AND ACTUARIAL SCIENCE, JARAMOGI OGINGA ODINGA \\ UNIVERSITY OF SCIENCE AND TECHNOLOGY, P. O. BOX 210-40601, BONDO-KENYA
}

Email: bnyaare@yahoo.com

\begin{abstract}
In this paper, a construction equivalent to "sum construction ", of BIBDs where BIBD is added to a BIBD that is automorphic to it is presented. The result is that we can get new BIBDs by forming the collection of a BIBD with its automorphic BIBDs. A new recursive technique has been developed for the construction of $t-\left(v, k, \lambda_{t}\right)$ designs. It has also clearly shown that every ${ }^{t-}$ design is also a BIB $(v, k, \lambda)$ design. Therefore, this construction technique also generates BIBDs. Therefore, this note presents an alternative method that is simpler and unified for the construction of BIBDs that are very important in the experimental designs. As it provides designs for different values of ${ }^{k}$, unlike many methods that provide designs for a single value of ${ }^{k}$. Moreso, it provides both Steiner and non- Steiner designs.
\end{abstract}

\section{Introduction}

The following are properties of ${ }^{t-\left(v, k, \lambda_{t}\right)}$ designs:

1. $\quad b k=\lambda_{1} v$

2. $\lambda_{t}(v-(t-1))=\lambda_{t-1}(k-(t-1))$

Indeed, number of ways of choosing $k$ out $v$ points is $\left(\begin{array}{l}v \\ k\end{array}\right)$. And any point will occur in $\left(\begin{array}{c}v-1 \\ k-1\end{array}\right)$ blocks. But we require each point to be incident to ${ }^{\lambda_{t}}$ blocks. Thus:

$$
b=\frac{\lambda_{1}\left(\begin{array}{l}
v \\
k
\end{array}\right)}{\left(\begin{array}{l}
v-1 \\
k-1
\end{array}\right)}
$$

Expanding R.H.S. we obtain:

$$
\begin{aligned}
b=\lambda_{1} \frac{v !}{k !(v-k) !} \div \frac{(v-1) !}{(k-1) !}(v-1-(k-1)) ! \\
=\lambda_{1} \frac{v !}{k !(v-k) !} \times \frac{(k-1) !(v-k) !}{(v-1) !} \\
=\lambda_{1} \frac{v !}{k !} \times \frac{(k-1) !}{(v-1) !} \\
=\lambda_{1} v \frac{(v-1) !}{k(k-1) !} \times \frac{(k-1) !}{(v-1) !} \\
=\lambda_{1} \frac{v}{k}
\end{aligned}
$$

Hence, $\quad b k=\lambda_{1} v$ as required

Similarly from the fact that ${ }^{t}$ points are incident to ${ }^{\lambda_{t}}$ blocks we have: 
Also

$$
\begin{aligned}
& b=\lambda_{t} \frac{\left(\begin{array}{l}
v \\
k
\end{array}\right)}{\left(\begin{array}{c}
v-t \\
k-t
\end{array}\right)} \\
& b=\lambda_{t-1} \frac{\left(\begin{array}{l}
v \\
k
\end{array}\right)}{\left(\begin{array}{l}
v-(t-1) \\
k-(t-1)
\end{array}\right)}
\end{aligned}
$$

Combining equation (i) and (ii) we have:

$$
\lambda_{t} \frac{\left(\begin{array}{c}
v \\
k
\end{array}\right)}{\left(\begin{array}{c}
v-t \\
k-t
\end{array}\right)}=\lambda_{t-1} \frac{\left(\begin{array}{c}
v \\
k
\end{array}\right)}{\left(\begin{array}{l}
v-(t-1) \\
k-(t-1)
\end{array}\right)}
$$

Expanding both sides we obtain:

$$
\begin{aligned}
\lambda_{t} \frac{v !}{k !(v-1) !} & \div \frac{(v-t) !}{(k-t) !(v-t-(k-t)) !} \\
& =\lambda_{t} \frac{v !}{k !(v-k) !} \div \frac{(v-(t-1)) !}{(k-(t-1)) !(v-(t-1)-(k-(t-1)) !}
\end{aligned}
$$

This reduces to:

$\lambda_{t} \frac{v !}{k !} \times \frac{(k-t) !}{(v-t) !}=\lambda_{t-1} \frac{v !}{k !} \times \frac{(k-(t-1) !}{(v-(t-1)) !}$

Multiplying both sides by $\frac{k !}{v !}$ we have

$\lambda_{t} \frac{(k-t) !}{(v-t) !}=\lambda_{t-1} \frac{(k-(t-1) !}{(v-(t-1) !}$

Expanding R.H.S. and simplifying we have:

$\lambda_{t} \frac{(k-t) !}{(v-t) !}=\frac{\lambda_{t-1}(k-(t-1))(k-t) !}{(v-(t-1))(v-t) !}$

Multiplying both sides by $\frac{(v-t) !}{(k-t) !}$ we have:

$\lambda_{t}=\frac{\lambda_{t-1}(k-(t-1)}{(v-(t-1))}$

Hence, $\quad \lambda_{t}(v-(t-1))=\lambda_{t-1}(k-(t-1)$

as required.

Equation (3.2) could be generalized as follows: starting with $t=6$ we have :

$\lambda_{6}(v-5)=\lambda_{5}(k-5)$

$\lambda_{5}(v-4)=\lambda_{4}(k-4), \quad t=5$

$\lambda_{4}(v-3)=\lambda_{3}(k-3), t=4$

$\lambda_{3}(v-2)=\lambda_{2}(k-2), t=3$

$\lambda_{2}(v-1)=\lambda_{1}(k-1), t=2$ 
Now:

$$
\begin{aligned}
& \lambda_{2}=\frac{\lambda_{1}(k-1)}{v-1} \\
& \lambda_{3}=\frac{\lambda_{1}(k-1)(k-2)}{(v-1)(v-2)} \\
& \lambda_{4}=\frac{\lambda_{1}(k-1)(k-2)(k-3)}{(v-1)(v-2)(v-3)} \\
& \lambda_{5}=\frac{\lambda_{1}(k-1)(k-2)(k-3)(k-4)}{(v-1)(v-2)(v-3)(v-4)} \\
& \lambda_{6}=\frac{\lambda_{1}(k-1)(k-2)(k-3)(k-4)(k-5)}{(v-1)(v-2)(v-3)(v-4)(v-5)} \\
& \lambda_{6}(v-1)(v-2)(v-3)(v-4)(v-5)=\lambda_{1}(k-1)(k-2)(k-3)(k-4)(k-5) \\
& \text { for } s=1,2 \ldots t-1 \text { weget } \\
& \lambda_{t}(v-(t-1))(v-(t-2))(v-(t-3)) \ldots(v-(t-s) \\
& \quad=\lambda_{t-s}(k-(t-1))(k-(t-2))(k-(t-3)) \ldots(k-(t-s)
\end{aligned}
$$

\section{MAIN RESULTS CONSTRUCTION OF 3- $(v, k, 1)$-DESIGN}

Here $t=3$ and $\lambda_{t}=1$ and putting this in equation (3.1.2) we have:

$v-2=\lambda_{2}(k-2)$

Hence

$\lambda_{2}=\frac{v-2}{k-2}$

Now when $t=2$ we have:

$\lambda_{2}(v-1)=\lambda_{1}(k-1)$

That is

$$
\frac{\lambda_{1}}{\lambda_{2}}=\frac{v-1}{k-1}
$$

This implies

$$
\lambda_{1}=\lambda_{2} \frac{(v-1)}{(k-1)} \quad ; \lambda_{1}=\propto(v-1)
$$

And

$$
\lambda_{2}=\lambda_{1} \frac{(k-1)}{(v-1)}
$$

Given that $\lambda_{1}, \lambda_{2}, v-1$ and $k-1$ are all integers and $\propto$ is a rational number which we will represent by $x / y$ where $x$ and $y$ are positive integers. Thus the equations (3.5) become:

$\lambda_{1}=\frac{x}{y}(v-1)$

$y \lambda_{1}=x(v-1)$

And

$\lambda_{2}=\frac{x}{y}(k-1)$ 


$$
y \lambda_{2}=x(k-1)
$$

Casel $\mathrm{x}=1$

Then (3.2.4) becomes

$y \lambda_{1}=v-1, \Rightarrow v=y \lambda_{1}+1$

And

$y \lambda_{2}=k-1, \Rightarrow k=y \lambda_{2}+1$

THEOREM 3.2 .1. IF $x=1$ and $\lambda_{2}-1 \equiv 0(\bmod y)$, where is an integer then there are only three non- trivial $3-(v, k, 1)$ designs which are: ${ }^{3-(8,4,1), 3-(22,6,1) \text { and } 3-(112,12,1)}$

Proof: Now from (3.2.1) we know

$\lambda_{2}=\frac{v-2}{k-2}$

But $v=y \lambda_{1}+1$ and $k=y \lambda_{2}+1$

Hence

$\lambda_{2}=\frac{y \lambda_{1}+1-2}{y \lambda_{2}+1-2}$

$=\frac{y \lambda_{1}-1}{y \lambda_{2}-1}$

This implies

$\lambda_{1}=\lambda^{2}{ }_{2}-\frac{\lambda_{2}-1}{y}$,

$v=y \lambda_{2}^{2}-\lambda_{2}+2$

And

$k=y \lambda_{2}+1$

For this design to be design $3-(v, k, 1)$ and from (3.1.1) it implies

$$
\left(\lambda^{2}{ }_{2}-\frac{\lambda_{2}-1}{y}\right)\left(y \lambda^{2}{ }_{2}-\lambda_{2}+2\right) \equiv 0 \bmod \left(y \lambda_{2}+1\right)
$$

That is

$\frac{\left(y \lambda_{2}^{2}-\lambda_{2}+1\right)\left(y \lambda^{2}{ }_{2}-\lambda_{2}+2\right)}{y\left(y \lambda_{2}+1\right)}$

Is a positive integer.

Expanding and simplifying we have:

$$
\begin{aligned}
& \frac{y^{2} \lambda_{2}^{4}-2 y \lambda_{2}^{3}+\lambda_{2}^{2}(3 y+1)-3 \lambda_{2}+2}{y^{2} \lambda_{2}+y} \\
& =\lambda_{2}^{3}-\frac{3 \lambda_{2}^{2}}{y}+\frac{\lambda_{2}(3 y+4)}{y^{2}}-\frac{6 y+4}{y^{3}} \text { rem } \frac{2 y^{2}+6 y+4}{y^{2}}
\end{aligned}
$$

Which can be written as:

$$
\lambda_{2}^{3}-\frac{3 \lambda_{2}^{2}}{y}+\frac{\lambda_{2}(3 y+4)}{y^{2}}-\frac{6 y+4}{y^{3}}+\frac{2 y^{2}+6 y+4}{y^{2}\left(y^{2} \lambda_{2}+y\right)}
$$

Now

$$
\frac{2 y^{2}+6 y+4}{y^{2}\left(y^{2} \lambda_{2}+y\right)}
$$


will be an integer if $y^{2}$ divides $6 y+4$.The only values for in which this is possible are 1 and 2 .

$$
\frac{2 y^{2}+6 y+4}{y^{2}\left(y^{2} \lambda_{2}+y\right)}=\frac{3}{2 \lambda_{2}+1}
$$

$$
\lambda_{2}>1
$$

In this case (3.2.6) is not an integer.

For $y=1 \quad(3.2 .6)$ is

$\frac{12}{\lambda_{2}+1}$

Thus both (3.2.5) and (3.2.6) will be integers if $\lambda_{2}$ takes the values $2,3,5$ and 11 .

The table below gives corresponding values of $k, \lambda_{1}, v$ and $b$.

Table 3.2.1. Case1; for $\mathrm{y}=1$ the possible cases of $3-(v, k, 1)$ designs

\begin{tabular}{|l|l|l|l|l|}
\hline$\lambda_{2}$ & $k$ & $\lambda_{1}$ & $v$ & $b$ \\
\hline 2 & 3 & 3 & 4 & 4 \\
\hline 3 & 4 & 7 & 8 & 14 \\
\hline 5 & 6 & 21 & 22 & 77 \\
\hline 11 & 12 & 111 & 112 & 1036 \\
\hline
\end{tabular}

The required $3-(v, k, 1)$ designs are; $3-(8,4,1), 3-(22,6,1)$, and $3-(112,12,1)$.

A $3-(4,3,1)$ is trivial given $t=k$, but it is required that $t<k$, hence is not included and our proof is completed

The above $3-(v, k, 1)$ designs are related with the following BIB designs: $2-(4,3,2), 2-(8,4,3), 2-(22,6,5), 2-(112,12,11)$. For example the configuration of

$2-(8,4,3)$ which is $3-(8,4,1)$ is:

$B 1(1,2,3,4), B 2(1,2,5,6), B 3(1,2,7,8), B 4(1,3,5,7), B 5(1,3,6,8), B 6(1,4,5,8), B 7(1,4,6,7), B 8(2,3,5,8), B 9(2,3,6$ $B 10(2,4,5,7), B 11(2,4,6,8), B 12(3,4,5,6), B 13(3,4,7,8), B 14(5,6,7,8)$.

3.2.1. Case 2, $y=1$

In this case (3.2.4) is

$$
\begin{aligned}
& \lambda_{1}=x(v-1), \quad \Rightarrow v=\frac{\lambda_{1}+x}{x} \\
& \lambda_{2}=x(k-1), \quad \Rightarrow k=\frac{\lambda_{2}+x}{x}
\end{aligned}
$$

Where is $x$ a positive integer and both $\lambda_{1}$ and $\lambda_{2}$ are divisible by $x$.

Now from (3.2.1)

$\lambda_{2}=\frac{v-2}{k-2}$

we get $\lambda_{1}, v$, and $k$ as follows;

$\lambda_{1}=\lambda^{2}{ }_{2}-x \lambda_{2}+x$,

$v=\frac{\lambda^{2}{ }_{2}-x \lambda_{2}+2 x}{x}$

and

$k=\frac{\lambda_{2}+x}{x}$ 
Using $b=\frac{\lambda_{1} v}{k}$. for this design to be $3-(v, k, 1)$ design then:

$\left(\lambda_{2}^{2}-x \lambda_{2}+x\right) \frac{\left(\lambda_{2}^{2}-x \lambda_{2}+2 x\right)}{x} \equiv 0 \bmod \left(\frac{\lambda_{2}+x}{x}\right)$

That is

$\frac{\left(\lambda_{2}^{2}-x \lambda_{2}+x\right)\left(\lambda^{2}{ }_{2}-x \lambda_{2}+2 x\right)}{\lambda_{2}+x}$

is a positive integer.

Expanding and dividing we will have:

$\lambda^{3}{ }_{2}-3 x \lambda^{2}{ }_{2}+\lambda_{2}\left(3 x+4 x^{2}\right)-\left(6 x^{2}+4 x^{3}\right)$ rem $2 x^{2}+6 x^{3}+4 x^{4}$

That is

$\lambda^{3}{ }_{2}-3 x \lambda^{2}{ }_{2}+\lambda_{2}\left(3 x+4 x^{2}\right)-\left(6 x^{2}+4 x^{3}\right)+\frac{2 x^{2}+6 x^{3}+4 x^{4}}{\lambda_{2}+x}$

Now (3.2.1.1) will be integer if

$$
\frac{2 x^{2}+6 x^{3}+4 x^{4}}{\lambda_{2}+x}
$$

For $x=2$ equation (3.2.1.2) is:

$\frac{120}{\lambda_{2}+2}, \lambda_{2}>2$

Thus $\lambda_{2}$ can take any of the following values $3,4,6,8,10,18,28,32,38,58$, andl18. But $\lambda_{2}$ must be divisible by 2 . So 3 is not a possibility. We give corresponding values of $k, \lambda_{1}, v$ and $b$ in the table below.

Table 3.2.1.1. Case 2; for $x=2$ the possible cases of $3-(v, k, 1)$ designs

\begin{tabular}{|l|l|l|l|l|}
\hline$\lambda_{2}$ & $k$ & $\lambda_{1}$ & $v$ & $b$ \\
\hline 4 & 3 & 10 & 6 & 20 \\
\hline 6 & 4 & 26 & 14 & 91 \\
\hline 8 & 5 & 50 & 26 & 260 \\
\hline 10 & 6 & 82 & 42 & 574 \\
\hline 18 & 10 & 290 & 146 & 4234 \\
\hline 22 & 12 & 442 & 222 & 8177 \\
\hline 28 & 15 & 730 & 366 & 17812 \\
\hline 38 & 20 & 1370 & 686 & 46991 \\
\hline 58 & 30 & 3250 & 1626 & 176150 \\
\hline 118 & 60 & 13690 & 6846 & 1562029 \\
\hline
\end{tabular}

The following designs $3-(6,3,1), 3-(14,4,1), 3-(26,5,1), 3-(42,6,1), 3-(146,10,1)$,

$3-(222,12,1), 3-(366,15,1), 3-(686,20),, 3-(1676,30,1)$ and $3-(6846,60,1)$. can then be obtain from $\operatorname{BIB}(v, k, \lambda)$ designs given below

$2-(6,3,4), 2-(14,4,6), 2-(26,5,8), 2-(42,6,10), 2-(146,10,18), 2-(222,12,22), 2-$

$(366,15,28), 2-(686,20,38), 2-(1626,30,58)$, and $2-(6846,60,118)$.

For $x=3$ equation (3.2.1.2) is

$\frac{504}{\lambda_{2}+3}, \lambda_{2}>3$

thus the possible values of $\lambda_{2}$ where, $\lambda_{2}$ is divisible by 3 are: $6,9,15,18,21,33,39,60,69,81,123,165,249$, and 501 .

We give corresponding values of $\lambda_{1}, v, k$, and $b$ in the table below. 
Table 3.2.1.2. Case 2; for $x=3$ the possible cases of $3-(v, k, 1)$ designs

\begin{tabular}{|l|l|l|l|l|}
\hline$\lambda_{2}$ & $k$ & $\lambda_{1}$ & $v$ & $b$ \\
\hline 6 & 3 & 21 & 8 & 56 \\
\hline 9 & 4 & 57 & 20 & 285 \\
\hline 15 & 6 & 183 & 62 & 1891 \\
\hline 18 & 7 & 273 & 92 & 3588 \\
\hline 21 & 8 & 381 & 128 & 6096 \\
\hline 33 & 12 & 993 & 332 & 27473 \\
\hline 39 & 14 & 1407 & 470 & 47235 \\
\hline 60 & 21 & 3423 & 1142 & 186146 \\
\hline 69 & 24 & 4557 & 1520 & 288610 \\
\hline 81 & 28 & 6321 & 2108 & 475881 \\
\hline 165 & 56 & 26733 & 8912 & 4254366 \\
\hline 249 & 84 & 61257 & 20420 & 14891285 \\
\hline 501 & 168 & 249501 & 83168 & 123514876 \\
\hline
\end{tabular}

Again we obtain $3-(v, k, 1)$ designs from BIB designs below:

$$
\begin{gathered}
2-(8,3,6), 2-(20,4,9), 2-(67,6,15), 2-(92,7,18), 2-(128,8,21), \\
2-(332,12,33), \ldots, \text { and } 2-(83168,168,501)
\end{gathered}
$$

For $x=4$, and using similar arguments as before we obtain possible values of $\lambda_{2}$ as follows: $8,12,16,20,28,32,36, \ldots$ which give the values of $\lambda_{1}, k, v$ and $b$ as follows:

Table 3.2.1.3. Case 2 ; for $x=4$ the possible cases of $3-(v, k, 1)$ designs

\begin{tabular}{|l|l|l|l|l|}
\hline$\lambda_{2}$ & $k$ & $\lambda_{1}$ & $\mathrm{~V}$ & $\mathrm{~B}$ \\
\hline 8 & 3 & 36 & 10 & 120 \\
\hline 12 & 4 & 100 & 26 & 650 \\
\hline 16 & 5 & 196 & 50 & 1960 \\
\hline 20 & 6 & 324 & 82 & 4428 \\
\hline 28 & 8 & 676 & 170 & 14365 \\
\hline 32 & 9 & 900 & 226 & 22600 \\
\hline 36 & 10 & 1156 & 290 & 33524 \\
\hline
\end{tabular}

The desired $3-(v, k, 1)$ designs are obtained from the following BIB designs:

$2-(10,3,8), 2-(26,4,12), 2-(50,5,16), 2-(82,6,20), 2-(170,8,28), 2-(226,9,32)$, and $2-(290,10,36)$

3.2.2. Case $3, x \neq y \quad x>1, y>1$

In this case (3.2.4) is

$$
\begin{aligned}
& \lambda_{1}=\frac{x}{y}(v-1) \quad \Longrightarrow v=\frac{y \lambda_{1}+x}{x} \\
& \lambda_{2}=\frac{x}{y}(k-1) \quad \Rightarrow k=\frac{y \lambda_{2}+x}{x}
\end{aligned}
$$

Where $x$ and $y$ are positive integers and $\lambda_{2}$ and $y$ are divisible by $x$.

Now from (3.2.1)

$\lambda_{2}=\frac{v-2}{k-2}$

We get $\lambda_{1}, v$, and $k$ as follows:

$\lambda_{1}=\frac{y \lambda_{2}^{2}-x \lambda_{2}+x}{y}$ 
$v=\frac{y \lambda_{2}^{2}-x \lambda_{2}+2 x}{x}$

And

$k=\frac{y \lambda_{2}+x}{x}$

Using $b=\frac{\lambda_{1} v}{k}$ for this to be $3-(v, k, 1)$ design then

$\frac{\left(y \lambda^{2}{ }_{2}-x \lambda_{2}+x\right)}{y}\left(\frac{y \lambda_{2}^{2}-x \lambda_{2}+2 x}{x}\right) \equiv 0 \bmod \left(\frac{y \lambda_{2}+x}{x}\right)$

That is

$\frac{\left(y \lambda^{2}{ }_{2}-x \lambda_{2}+x\right)\left(y \lambda_{2}^{2}-x \lambda_{2}+2 x\right)}{y\left(y \lambda_{2}+x\right)}$

Is a positive integer.

Expanding and dividing we will obtain

$$
y \lambda^{3}{ }_{2}-3 x \lambda_{2}^{2}+\frac{\lambda_{2}^{2}\left(3 x y+4 x^{2}\right)}{y}-\frac{\left(6 x^{2} y+4 x^{3}\right)}{y^{2}} \operatorname{rem} \frac{2 x^{2} y^{2}+6 x^{3} y+4 x^{4}}{y^{2}}
$$

That is:

$y \lambda^{3}{ }_{2}-3 x \lambda_{2}^{2}+\frac{\lambda^{2}{ }_{2}\left(3 x y+4 x^{2}\right)}{y}-\frac{\left(6 x^{2} y+4 x^{3}\right)}{y^{2}}+\frac{2 x^{2} y^{2}+6 x^{3} y+4 x^{4}}{y^{3}\left(y \lambda_{2}+x\right)}$

Now (3.2.2.1) will be an integer if

$$
\frac{2 x^{2} y^{2}+6 x^{3} y+4 x^{4}}{y^{3}\left(y \lambda_{2}+x\right)}=\frac{2 x^{2}\left(1+\frac{3 x}{y}+2 \frac{x^{2}}{y^{2}}\right)}{y\left(y \lambda_{2}+x\right)}
$$

Is an integer.

For $x=3$ and $y=2$,

Equation (3.2.2.2) in this case is:

$\frac{90}{2 \lambda_{2}+3}$

In this case there is only one non-trivial $3-(v, k, 1)$ which we get as follows: the possible values of $\lambda_{2}$ are 3 or 21 .

We give corresponding values of $\lambda_{1}, k, v$, and $b$ in the table below.

Table 3.2.2.1. Case 3; for $x=3$ and $y=2$ the possible cases of $3-(v, k, 1)$ designs

\begin{tabular}{|l|l|l|l|l|}
\hline$\lambda_{2}$ & $k$ & $\lambda_{1}$ & $v$ & $b$ \\
\hline 3 & 3 & 6 & 5 & 10 \\
\hline 21 & 15 & 411 & 275 & 7535 \\
\hline
\end{tabular}

For $x=5$, and $y=2$

In this case the equation (3.2.2.2) becomes:

$\frac{525}{2 \lambda_{2}+5}$

The corresponding values of $\lambda_{1}, \lambda_{2}, k, v$, and $b$ are as in the table below: 
Table 3.2.2.2. Case 3; for $x=5$ and $y=2$ the possible cases of $3-(v, k, 1)$ designs

\begin{tabular}{|l|l|l|l|l|}
\hline$\lambda_{2}$ & $k$ & $\lambda_{1}$ & $v$ & $b$ \\
\hline 5 & 3 & 15 & 7 & 35 \\
\hline 15 & 7 & 190 & 77 & 2090 \\
\hline 35 & 15 & 1140 & 457 & 34732 \\
\hline 85 & 35 & 7015 & 2807 & 562603 \\
\hline
\end{tabular}

For $x=7$, and $y=2(3.2 .2 .2)$ becomes

\section{4}

$\overline{2 \lambda_{2}+7}$

And we get the following table.

Table 3.2.2.3. Case 3; for $x=7$ and $y=2$ the possible cases of $3-(v, k, 1)$ designs

\begin{tabular}{|l|l|l|l|l|}
\hline$\lambda_{2}$ & $k$ & $\lambda_{1}$ & $v$ & $b$ \\
\hline 7 & 3 & 28 & 9 & 84 \\
\hline 21 & 7 & 371 & 107 & 5671 \\
\hline 217 & 63 & 46333 & 13239 & 9736549 \\
\hline
\end{tabular}

\section{CONSTRUCTION OF $t-(v, k, 1)$ DESIGNS WITH $t=3$ and $\lambda_{t} \geq 1$}

In this section we extend the work in Onyango (2010) by constructing 3 - designs with, that $\lambda_{t} \geq 1$ is for general index and Steiner 4 - designs

\subsubsection{CONSTRUCTION OF $3-(v, k, 1)$}

Here $t=3, \lambda_{t}=c$ and from (3.1.2) we have:

$\lambda_{2}=\frac{c(v-2)}{k-2} \quad$ for $\lambda_{2}>1$

Now when $t=2$ we have:

$\frac{\lambda_{1}}{\lambda_{2}}=\frac{v-1}{k-1}$

Which implies;

$$
\lambda_{1}=\frac{\lambda_{2}(v-1)}{k-1} ; \Rightarrow \lambda_{1}=\propto(v-1)
$$

And

$$
\lambda_{2}=\frac{\lambda_{1}(k-1)}{v-1} ; \Rightarrow \lambda_{2}=\stackrel{\prime}{=} \propto(k-1)
$$

As mentioned earlier, $\propto$ is a rational number since $\lambda_{1}, \lambda_{2}, c, v-1$ and $k-1$ are all positive integers hence, we will represent it by $\frac{x}{y}$ where $x$ and $y$ are positive integers.

3.3.2. CASE $I, x=1$

Then (3.3.1.3) becomes:

$\lambda_{1}=\frac{v-1}{y}, \Rightarrow v=y \lambda_{1}+1$

And

$\lambda_{2}=\frac{k-1}{y}, \Rightarrow k=y \lambda_{2}+1$

Now from (3.3.1.1) we have: 
$\lambda_{2}=\frac{c(v-2)}{k-2}$

But

$$
v=y \lambda_{1}+1 \text {, and } k=y \lambda_{2}+1
$$

Hence

$$
\lambda_{2}=\frac{c\left(y \lambda_{1}-1\right)}{y \lambda_{2}-1}
$$

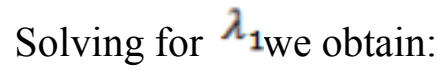

$$
\lambda_{1}=\frac{y \lambda_{2}^{2}-\lambda_{2}+c}{c y}
$$

And

$$
v=\frac{y \lambda_{2}^{2}-\lambda_{2}+2 c}{c}
$$

For this design to be $3-(v, k, c)$ design and from $b k=v \lambda_{1}$; it implies

$$
\frac{\left(y \lambda_{2}^{2}-\lambda_{2}+c\right)}{c y}\left(\frac{y \lambda_{2}^{2}-\lambda_{2}+2 c}{c}\right) \equiv \bmod \left(y \lambda_{2}+1\right)
$$

That is,

$$
\frac{\left(y \lambda_{2}^{2}-\lambda_{2}+c\right)\left(y \lambda_{2}^{2}-\lambda_{2}+2 c\right)}{c^{2} y\left(y \lambda_{2}+1\right)}
$$

Is a positive integer.

Expanding and dividing we have:

$$
\frac{\lambda_{2}^{3}}{c^{2}}-\frac{3 \lambda^{2}{ }_{2}}{c^{2} y}+\frac{\lambda_{2}(3 c y+4)}{c^{2} y^{2}}-\frac{(6 c y+4)}{c^{2} y^{3}}+\frac{2 c^{2} y^{2}+6 c y+4}{c^{2} y^{2}\left(y^{2} \lambda_{2}+y\right)}
$$

Now (3.3.2.1) will be an integer if $c^{2} y^{2}$ divides $6 c y+4$

For $c=2$, that is $\lambda_{t}=2$

The only possible values for $y$ in which this is possible are 1 and 2. Using;

$$
\frac{2 c^{2} y^{2}+6 c y+4}{c^{2} y^{2}\left(y^{2} \lambda_{2}+y\right)}
$$

For $y=2$ we have:

$$
\frac{15}{8\left(2 \lambda_{2}+1\right)}
$$

In this case (3.3.2.2.) is not an integer.

For $y=1$ the equation (3.3.2.2) becomes:

$$
\frac{6}{\lambda_{2}+1}
$$

Thus (3.3.2.2) will be an integer if $\lambda_{2}$ takes only of the following values; 2 and 5 .

The table below gives corresponding values of $\lambda_{1}, k, v$ and $b$

Table 3.2.2.1. Case 1 ; for $y=1$ and $c=2$ the possible cases of $3-(v, k, 1)$ designs

\begin{tabular}{|l|l|l|l|l|}
\hline$\lambda_{2}$ & $k$ & $\lambda_{1}$ & $v$ & $b$ \\
\hline 2 & 3 & 2 & 3 & 2 \\
\hline 5 & 6 & 11 & 12 & 22 \\
\hline
\end{tabular}


The first $3-(3,3,2)$ does not exist hence we have only one $3-(v, k, c)$ design in this case. This $3-(12,6,2)$ is identified with this BIB design $B(12,6,5)$.

3.3.2. CASE I, $y=1$

In this case (3.3.1.3) becomes:

$\lambda_{1}=x(v-1), \quad \Rightarrow v=\frac{\lambda_{1}+x}{x}$

And

$\lambda_{2}=x(k-1), \quad \Rightarrow k=\frac{\lambda_{2}+x}{x}$

Now from (3.3.1.1) we have:

$\lambda_{2}=\frac{c\left(\lambda_{1}-x\right)}{\lambda_{2}-x}$

Solving for $\lambda_{1}$ we have:

$\lambda_{1}=\frac{\lambda_{2}^{2}-x \lambda_{2}+c x}{c}$

Now

$v=\frac{\lambda_{2}^{2}-x \lambda_{2}+2 c x}{c x}$

Using similar argument as before:

$\frac{\left(\lambda_{2}^{2}-x \lambda_{2}+x c\right)\left(\lambda_{2}^{2}-x \lambda_{2}+2 c x\right)}{c^{2}\left(\lambda_{2}+x\right)}$

Will be integer if

$\frac{2 c^{2} x^{2}+6 c x^{3}+4 x^{4}}{c^{2}\left(\lambda_{2}+x\right)}$

Is an integer. Note that when $x=1$ we get results of the previous case.

For $c=2$ and $x=2$ equation (3.3.3.2) is

$\frac{48}{\lambda_{2}+2}, \quad \lambda_{2}>2$

We give the corresponding values of $\lambda_{2}, \lambda_{1}, v, k$ and $b$ in the table below;

Table 3.3.3.1. Case 2 ; for $x=2$ and $c=2$ the possible cases of $3-(v, k, 1)$ designs

\begin{tabular}{|l|l|l|l|l|}
\hline$\lambda_{2}$ & $k$ & $\lambda_{1}$ & $v$ & $b$ \\
\hline 4 & 3 & 6 & 4 & 8 \\
\hline 6 & 4 & 14 & 8 & 28 \\
\hline 10 & 6 & 42 & 22 & 154 \\
\hline 14 & 8 & 86 & 44 & 473 \\
\hline 22 & 12 & 222 & 112 & 2072 \\
\hline 46 & 24 & 1014 & 508 & 21463 \\
\hline
\end{tabular}


The following designs;

$3-(4,3,2), 3-(8,4,2), 3-(22,6,2), 3-(44,8,2), 3-(112,12,2)$, and $3-(508,24,2)$ can be obtained from $B I B(v, k, \lambda)$ designs given| below:

$B(4,3,4), B(8,4,6), B(22,6,10), B(44,8,14), B(112,12,22)$, and $B(508,24,46)$.

For $c=2, x=3$ and using similar arguments as before we give the values of $\lambda_{2}, \lambda_{1}, v, k$ and $b$ as in the table below:

Table 3.3.3.2. Case 2; for $x=3$ and $c=2$ the possible cases of $3-(v, k, 1)$ designs

\begin{tabular}{|l|l|l|l|l|}
\hline$\lambda_{2}$ & $k$ & $\lambda_{1}$ & $\mathrm{~V}$ & $\mathrm{~B}$ \\
\hline 6 & 3 & 12 & 5 & 20 \\
\hline 12 & 5 & 57 & 20 & 228 \\
\hline 15 & 6 & 93 & 32 & 496 \\
\hline 27 & 10 & 327 & 110 & 3597 \\
\hline
\end{tabular}

We get the desired $3-(v, k, c)$ designs from BIB designs below:

$B(5,3,6), B(20,5,12), B(32,6,15)$ and $(110,10,27)$.

For $c=2, x=4$ and using the same methods we give the table of values of; $\lambda_{2}, \lambda_{1}, k, v$ and $b$ as follows:

Table 3.3.3.3. Case 2; for $x=4$ and $c=2$ the possible cases of $3-(v, k, 1)$ designs

\begin{tabular}{|l|l|l|l|l|}
\hline$\lambda_{2}$ & $k$ & $\lambda_{1}$ & $v$ & $b$ \\
\hline 8 & 3 & 20 & 6 & 40 \\
\hline 12 & 4 & 52 & 14 & 182 \\
\hline 16 & 5 & 100 & 26 & 520 \\
\hline 20 & 6 & 168 & 42 & 1126 \\
\hline 36 & 10 & 580 & 146 & 8468 \\
\hline 44 & 12 & 884 & 222 & 16354 \\
\hline 56 & 15 & 1460 & 366 & 35624 \\
\hline
\end{tabular}

Similarly, for $c=2, x=5$ the values of $\lambda_{1}, \lambda_{2}, k, v$ and $b$ we give them as in the table below:

Table 3.3.3.4. Case 2; for $x=5$ and $c=2$ the possible cases of $3-(v, k, 1)$ designs

\begin{tabular}{|l|l|l|l|l|}
\hline$\lambda_{2}$ & $k$ & $\lambda_{1}$ & $v$ & $b$ \\
\hline 10 & 3 & 30 & 7 & 70 \\
\hline 20 & 5 & 155 & 32 & 992 \\
\hline 25 & 6 & 255 & 52 & 2210 \\
\hline 30 & 7 & 380 & 77 & 4180 \\
\hline 65 & 14 & 1955 & 392 & 54740 \\
\hline
\end{tabular}

Now, for $c=3, x=3$ and $x=6$ and applying the same methods, we give values of $\lambda_{2}, \lambda_{1}, v, k$ and $b$ in the tables below respectively.

Table 3.3.3.5. Case 2 ; for $x=3$ and $c=3$ the possible cases of $3-(v, k, 1)$ designs

\begin{tabular}{|l|l|l|l|l|}
\hline$\lambda_{2}$ & $k$ & $\lambda_{1}$ & $v$ & $b$ \\
\hline 6 & 3 & 9 & 4 & 12 \\
\hline 9 & 4 & 21 & 8 & 42 \\
\hline 15 & 6 & 63 & 22 & 231 \\
\hline 24 & 9 & 171 & 58 & 1102 \\
\hline 51 & 18 & 819 & 274 & 12467 \\
\hline
\end{tabular}


Table 3.3.3.6. Case 2; for $x=6$ and $c=3$ the possible cases of $3-(v, k, 1)$ designs

\begin{tabular}{|l|l|l|l|l|}
\hline$\lambda_{2}$ & $k$ & $\lambda_{1}$ & $v$ & $b$ \\
\hline 12 & 3 & 30 & 6 & 60 \\
\hline 18 & 4 & 78 & 14 & 273 \\
\hline 24 & 5 & 150 & 26 & 780 \\
\hline 30 & 6 & 246 & 42 & 1722 \\
\hline
\end{tabular}

Also for $c=4, x=2, x=4$ and $x=6$ and using similar arguments, we give values of $\lambda_{2}, \lambda_{1}, v, k$, and $b$ in the tables below respectively.

Table 3.3.3.7. Case 2; for $x=2$ and $c=4$ the possible cases of $3-(v, k, 1)$ designs

\begin{tabular}{|l|l|l|l|l|}
\hline$\lambda_{2}$ & $k$ & $\lambda_{1}$ & $v$ & $b$ \\
\hline 4 & 3 & 4 & 3 & 4 \\
\hline 6 & 4 & 8 & 5 & 10 \\
\hline 10 & 6 & 22 & 12 & 44 \\
\hline 22 & 12 & 112 & 57 & 532 \\
\hline
\end{tabular}

The first $3-(3,3,4)$ design is trivial.

Table 3.3.3.8. Case 2; for $x=4$ and $c=4$ the possible cases of $3-(v, k, 1)$ designs

\begin{tabular}{|l|l|l|l|l|}
\hline$\lambda_{2}$ & $k$ & $\lambda_{1}$ & $v$ & $b$ \\
\hline 12 & 4 & 28 & 8 & 56 \\
\hline 20 & 6 & 84 & 22 & 308 \\
\hline 28 & 8 & 172 & 44 & 946 \\
\hline
\end{tabular}

Table 3.3.3.9. Case 2 ; for $x=6$ and $c=4$ the possible cases of $3-(v, k, 1)$ designs

\begin{tabular}{|l|l|l|l|l|}
\hline$\lambda_{2}$ & $k$ & $\lambda_{1}$ & $v$ & $b$ \\
\hline 18 & 4 & 60 & 11 & 165 \\
\hline 24 & 5 & 114 & 20 & 456 \\
\hline 30 & 6 & 186 & 32 & 992 \\
\hline
\end{tabular}

When $c=5, x=5$ and $x=10$ and using similar methods the values of $\lambda_{2}, \lambda_{1}, v, k$ and $b$ we give them in the tables below respectively.

Table 3.3.3.10. Case 2 ; for $x=5$ and $c=5$ the possible cases of $3-(v, k, 1)$ designs

\begin{tabular}{|l|l|l|l|l|}
\hline$\lambda_{2}$ & $k$ & $\lambda_{1}$ & $v$ & $b$ \\
\hline 15 & 4 & 35 & 8 & 70 \\
\hline 20 & 5 & 65 & 14 & 182 \\
\hline 25 & 6 & 105 & 22 & 385 \\
\hline 45 & 10 & 365 & 74 & 2701 \\
\hline
\end{tabular}

Table 3.3.3.11. Case 2; for $x=10$ and $c=5$ the possible cases of $3-(v, k, 1)$ designs

\begin{tabular}{|l|l|l|l|l|}
\hline$\lambda_{2}$ & $k$ & $\lambda_{1}$ & $v$ & $b$ \\
\hline 30 & 4 & 130 & 14 & 455 \\
\hline 40 & 5 & 250 & 26 & 1300 \\
\hline 50 & 6 & 410 & 42 & 2870 \\
\hline
\end{tabular}

or $c=6, x=3$ and $x=6$ we get the following tables respectively. 


\section{Conclusions:}

In this study a new recursive technique has been developed for the construction of $t-\left(v, k, \lambda_{t}\right)$ designs. It has also clearly shown that every $t$-design is also a BIB $\left(v, k, \lambda_{t}\right)$ design. Therefore, this construction technique also generates BIBDs. Thus, the study has presented an alternative method that is simpler and unified for the construction of BIBDs that are very important in the experimental designs. As it provides designs for different values of $k$, unlike many methods that provide designs for a single value of $k$. Moreso, it provides both Steiner and non-Steiner designs.

\section{Recommendation:}

Although this study has provided a technique for the construction of $t$-designs, it is still clear that construction method of $t$-designs is not known in general. In fact, it is not clear how one might construct $t$ - designs with arbitrary block size. We therefore invite researchers to come up with "additive theorems "for this construction to make it general for any value of as this may bring in new techniques and ideas. There is also need for obtaining a theorem which would give all values of $\mathrm{x}$ and $\mathrm{y}$ for the case three in this construction in order to see new Steiner $t$-designs. Lastly, if there is a computer package that could be incorporated in the method to aid in calculations

\section{REFERENCES:}

[1] Adhikari, B. (1967). On the symmetric differences of pairs of blocks of incomplete block designs, Calcutta stat. Assoc. Bull 16, 45-48.

[2] Anderson, I. (1989). A first Course in Combinatorial Mathematics second edition, Oxford University Press, New York.

[3] Blanchard, J.L (1995a). A construction for Steiner 3-designs, Journal of combinatorial Theory A, 71, 60-67.

[4] Blanchard, J.L. (1995b). An extension theorem for Steiner systems, Discrete Mathematics 141, no. 1-3, 23-35.

[5] Blanchard, J. L (1995c).A construction for orthogonal arrays with strength $t \geq 3$, Discrete math 137 , no. $1-3,35-44$.

[6] Bogart, K.P. (1990). Introductory Combinatorics second edition, Harcourt Brace Jovanovich, Inc. Orlando, Florida.

[7] Bose, R.C. (1950). A note on orthogonal arrays, Annals of math.stat.21, 304-305

[8] Cameron, P. J., Maimani, H. R., Omidi, G.R., and Tayfeh-Rezaie, B. (2006). 3-designs PGL (2, q), Discrete Mathematics, 306, vol.23, 3063-3073.

[9] Colbourn, C.J.et al. (2002). Orthogonal arrays of strength three from regular 3-wise balanced designs.

[10]Chowla,S and Ryser,H.J.(1950). Combinatorial problems. Canadian journal of mathematics.2, 93-9

[11]Dinitz, J. H., \& Stinson, D. R. (1992). Contemporary Design Theory: A collection of surveys, Wiley-Interscience.

[12]Fisher R.A. (1940). An examination of the difference possible solutions of a problem in incomplete blocks. Annals of Engenics 10, 52-75.

[13]Hanan, H. (1960). On quadruple systems, Canadian Journal of mathematics, 15, 145-157.

[14] Hanani, H. (1963). On some tactical configurations, Canadian Journal of mathematics, 15, 702722 